\title{
Protozoarios parásitos de importancia en salud pública transportados por Musca domestica Linnaeus en Lima, Perú
}

\section{Parasite protozoa of importance in public health picked up by Musca domestica Linnaeus in Lima, Peru}

\author{
Martín Cárdenas y Rosa Martínez*
}

Presentado: $16 / 11 / 2004$

Aceptado: $\quad 02 / 12 / 2004$

\section{Resumen}

El presente trabajo investiga los protozoarios parásitos de importancia para la salud pública transportados por Musca domestica. El estudio se llevó a cabo entre los meses de junio y diciembre de 1998, en los distritos de Comas y San Juan de Lurigancho de la provincia de Lima. Se estudiaron 3014 moscas colectadas en la basura acumulada en la vía pública de los pueblos jóvenes de los distritos mencionados. Las capturas se agruparon en lotes constituidos por un promedio de 30 moscas. Se estudiaron un total de 100 lotes. Las moscas fueron disecadas y procesadas con agua destilada estéril para obtener un macerado intestinal. Para la observación de los parásitos se utilizó el método directo simple y la coloración tricrómica de Gomori ácido resistente modificado. Se encontraron los siguientes protozoarios que parasitan al hombre: Blastocystis hominis $(9,0 \%)$, Giardia lamblia (3,0\%), Cryptosporidium sp. $(2,0 \%)$, Cyclospora cayetanensis $(1,0 \%)$, lodamoeba bütschlii $(17,0 \%)$, Endolimax nana (5,0\%) y Chilomastix mesnili (3,0\%). Se reporta por primera vez para el Perú a B. hominis y Cyclospora cayetanensis en M. domestica.

Palabras claves: Musca domestica, protozoarios, salud pública, Lima.

\section{Abstract}

At the present work we had investigated protozoa parasite picked up by Musca domestica. Surveys were carry out between June and December 1998 in districts of Lima (Comas and San Juan de Lurigancho). A total of 3014 flies were collected around garbage accumulated in the streets. Each survey were considered a lot with a mean of 30 flies. A total of 100 lots were studied. The flies were dissected and processed with sterilised distilled water for obtain the intestinal macerated. Observations were made by the simple direct method and with Gomori trichromic stain modified acid-fast. The following protozoa parasites of human were found: Blastocystis hominis $(9,0 \%)$, Giardia lamblia $(3,0 \%)$, Cryptosporidium sp. (2,0\%), Cyclospora cayetanensis (1,0\%), lodamoeba bütschlii (17,0\%), Endolimax nana $(5,0 \%)$ and Chilomastix mesnili $(3,0 \%)$. B. hominis y Cyclospora cayetanensis were reported for the first time in M. domestica from Peru.

Keywords: Musca domestica, protozoa, Public Health. Lima.

\section{Introducción}

Musca domestica Linnaeus es un insecto de amplia distribución mundial y frecuentemente asociado al hombre. Exceptuando el Ártico, el Antártico y áreas de extrema altitud, la mosca se ha adaptado con éxito a todas las condiciones, predominando en las habitaciones humanas y sus alrededores (OPS, 1962). Varios facto-

\footnotetext{
(*)Laboratorio de Parasitología de Fauna Silvestre. Facultad de Ciencias Biológicas, Universidad Nacional Mayor de San Marcos. Av. Venezuela cuadra 34 s/n, Lima.
}

E-mail: Rosa Martínez rmartinezr@unmsm.edu.pe res biológicos, entre ellos la adaptación al medio ambiente, capacidad de dispersión y alto poder reproductivo aseguran su población durante todo el año (Moreno, 1980; Keiding, 1987).

La mosca doméstica puede transportar organismos patógenos sobre la superficie corporal, así como en el interior de la cavidad intestinal y diseminarlos en las regurgitaciones y en las heces (Keiding, 1987).

Las condiciones inadecuadas de las viviendas, el mal saneamiento básico y la situación socioeconómica deficiente son factores que 
contribuyen a la aparición y propagación de las moscas (OPS, 1962; Camac, 1992); así mismo, los residuos sólidos urbanos son considerados como determinantes de la estructura epidemiológica de la comunidad, actuando sinérgicamente al lado de otros factores ambientales. Por otro lado la acumulación de basura tanto en los domicilios como en la vía pública constituyen una fuente importante para la reproducción y desarrollo de $M$. domestica (Keiding,1987).

En el ámbito mundial la incriminación de las moscas como vectores de agentes patógenos se ha mantenido desde hace muchos años. Coutihno et al. (1957) realizaron una revisión sobre la mosca doméstica como transmisor mecánico de formas parasitarias del hombre. Sin embargo, actualmente la mayoría de informes con relación a las enfermedades transmitidas por insectos vectores dan poca importancia a $M$. domestica, y en el Perú no se ha investigado sobre la real magnitud de la transmisión de enteroparásitos por este vector mecánico.

Por otro lado, la literatura nacional referida al transporte de formas parasitarias por $M$. domestica que pueden infectar al ser humano es muy escasa (Guillén de Tantaleán et al., 1984; Zarate y Mejía, 1995; Gamarra y Ocampos, 1996; Custodio y Murga, 1997; Miranda y Martínez, 1997). Por estas consideraciones, hemos creido necesario estudiar el papel de la mosca doméstica en el transporte de protozoarios, en especial de aquellos que son patógenos y poco estudiados.

\section{Material y métodos}

Entre los meses de junio y diciembre de 1998, se colectaron un total de 3014 especímenes de Musca domestica procedentes de los basurales formados en la vía pública de pueblos jóvenes de los distritos de Comas y San Juan de Lurigancho de la provincia de Lima. Con estos insectos se formaron 100 lotes cada uno de los cuales constituido por aproximadamente 30 moscas.

La captura de los insectos se efectúo utilizando una red entomológica $(15 \mathrm{~cm}$ de diá- metro) confeccionada para tal fin, luego se colocaron en bolsas de polietileno, las que fueron debidamente rotuladas con la procedencia y cantidad colectada.

Las moscas fueron sacrificadas en frascos letales utilizando cloroformo. La disección se realizó sobre láminas portaobjeto; los intestinos fueron colocados en viales que contenían agua destilada estéril, los cuales se agitaron durante 3 minutos y se dejaron sedimentar. Parte del sedimento se colocó sobre una lámina portaobjeto, se mezcló con una o dos gotas del fijador alcohol polivinílico, se homogenizó y se dejó secar entre 48 a 72 horas. Posteriormente estas láminas fueron coloreadas aplicando el método tricrómico de Gomori (Tantaleán, 1998). La otra parte del sedimento se depositó en viales que contenían una pequeña cantidad de solución de bicromato de potasio al 2,5\%, para la tinción de ooquistes de coccidios siguiendo el método de ZiehlNeelsen modificado por Kinyoun (Tantaleán, 1998). Adicionalmente, a uno o dos especímenes de cada lote se les disecó el intestino y el contenido intestinal fue procesado para observación directa utilizando unas gotas de solución salina.

\section{Resultados y discusión}

Se encontraron 7 especies de protozoarios parásitos que pueden infectar al hombre: Blastocystis hominis, Giardia lamblia, Cryptosporidium sp, Cyclospora cayetanensis, Iodamoeba bütschlii, Endolimax nana y Chilomastix mesnili. Además se encontraron 17 lotes con quistes de $I$. bütschlii y 9 con trofozoitos de $B$. hominis correspondiendo éstos a las mayores frecuencias; del mismo modo, se observó dos lotes con ooquiste de Cryptosporidium sp. y uno con ooquiste de Cyclospora cayetanensis (Tabla 1).

En la localidad de Año Nuevo, distrito de Comas, se observó la mayor variedad de protozoarios parásitos (4 especies), mientras que en la localidad de Huáscar, en San Juan de Lurigancho, se observaron toda la variedad de protozoarios antes mencionados. Estos distritos 
Tabla 1. Protozoarios parásitos del hombre encontrados en Musca domestica, colectadas en dos distritos de Lima, Comas y San Juan de Lurigancho, entre junio y diciembre de 1998. Se indican las localidades de colecta, el número de lotes, cada lote con un promedio de 30 moscas examinadas y el número de lotes con presencia de protozoarios parásitos (positivos).

\begin{tabular}{|c|c|c|c|c|c|c|c|c|c|c|c|c|c|c|c|}
\hline & & \multicolumn{2}{|c|}{ 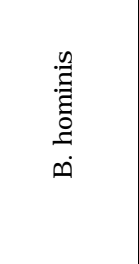 } & \multicolumn{2}{|c|}{ 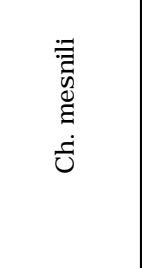 } & \multicolumn{2}{|c|}{ 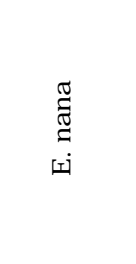 } & \multicolumn{2}{|c|}{ 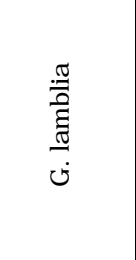 } & \multicolumn{2}{|c|}{ 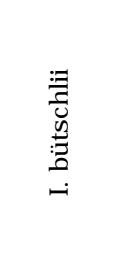 } & \multicolumn{2}{|c|}{ 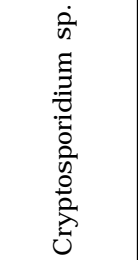 } & \multicolumn{2}{|c|}{ 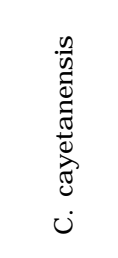 } \\
\hline Localidad & 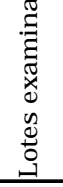 & $\begin{array}{l}D^{n} \\
\stackrel{7}{n=0} \\
0 \\
0\end{array}$ & de & 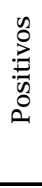 & de & $\begin{array}{l}0 \\
\stackrel{0}{0} \\
0 \\
0 \\
0\end{array}$ & $d^{0}$ & 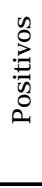 & do & $\begin{array}{l}0 \\
0 \\
\stackrel{⿹}{n} \\
0 \\
0\end{array}$ & de & 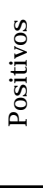 & $\partial^{0}$ & $\begin{array}{l}0 \\
0 \\
\stackrel{7}{0} \\
0 \\
0\end{array}$ & $d^{0}$ \\
\hline \multicolumn{16}{|l|}{ Comas } \\
\hline Collique & 7 & - & - & - & - & - & - & - & - & 1 & 14,3 & - & - & - & - \\
\hline Año Nuevo & 8 & 2 & 25,0 & 1 & 12,5 & 2 & 25,0 & - & - & 4 & 50,0 & - & - & - & - \\
\hline San Juan Bautista & 8 & - & - & - & - & - & - & - & - & 1 & 12,5 & - & - & - & - \\
\hline Sinchi roca & 10 & 2 & 20,0 & - & - & 1 & 10,0 & 1 & 10,0 & - & & - & - & - & - \\
\hline La Pascana & 9 & 1 & 11,1 & - & - & - & - & - & - & 4 & 44,4 & - & - & - & - \\
\hline La Merced & 8 & - & - & - & - & - & - & - & - & 1 & 12,5 & - & - & - & - \\
\hline \multicolumn{16}{|c|}{ San Juan de Lurigancho } \\
\hline Huascar i & 13 & 2 & 15,4 & 1 & 7,7 & 1 & 7,7 & 1 & 7,7 & 2 & 15,4 & 2 & 15,4 & 1 & 7,7 \\
\hline Huascar ii & 8 & 1 & 12,5 & - & - & - & - & - & - & 1 & 12,5 & - & - & - & - \\
\hline 10 de Febrero & 7 & - & - & - & - & - & - & 1 & 14,3 & 1 & 14,3 & - & - & - & - \\
\hline Canto rey & 6 & - & - & - & - & 1 & 16,7 & - & - & 1 & 16,7 & - & - & - & - \\
\hline Las flores & 8 & - & - & 1 & 12,5 & - & - & - & - & - & - & - & - & - & - \\
\hline Jesus oropeza & 8 & 1 & 12,5 & - & - & - & - & - & - & 1 & 12,5 & - & - & - & - \\
\hline Total & 100 & 9 & 9,0 & 3 & 3,0 & 5 & 5,0 & 3 & 3,0 & 17 & 17,0 & 2 & 2,0 & 1 & 1,0 \\
\hline
\end{tabular}

presentan condiciones ambientales similares y durante el tiempo en que se realizó la captura de moscas la formación de basurales fue frecuente.

B. hominis y Cyclospora cayetanenesis. son reportados por primera vez para el Perú en $M$. domestica. Estos protozoarios afectan mayormente a pobladores de zonas marginales en nuestro país, produciendo cuadros clínicos diversos entre los que destacan el dolor abdominal, náuseas, anorexia, fatiga, la diarrea y perdida de peso (Acosta, 1997; Colomina y Villar, 1997). En la presente investigación encontramos 9 lotes $(9,0 \%)$ con trofozoitos de $B$. hominis y un lote con ooquiste de Cyclospora cayetanensis. Ambos protozoarios junto con $G$. lamblia y Cryptosporidium sp. son considerados como los más patógenos para el hombre. La presencia de ellos se explicaría por el hábito del insecto de vivir en contacto y alimen- tándose de materia en descomposición y excretas del hombre y de animales.

Nuestros resultados son coincidentes parcialmente con investigaciones anteriores. Así Guillén de Tantaleán et al. (1984), luego de analizar un total de 1740 moscas capturadas en basurales, viviendas y en un establo de la Provincia Constitucional del Callao, hallaron quistes de Endolimax nana, Entamoeba coli, Iodamoeba bütschlii y huevos de Ascaris lumbricoides. Gamarra y Ocampos (1996) señalaron el transporte de E. coli, E. nana, G. lamblia, A. lumbricoides, Trichuris trichura, Hymenolepis nana y Taenia sp. Custodio y Murga(1997), al investigar 100 ejemplares de $M$. domestica mencionan el hallazgo de E. coli, Diphyllobothrium sp. y de A. lumbricoides. Es importante señalar que no obstante haber estudiado un número consi- 
derable de moscas, no hemos encontrado huevos de céstodos o nemátodos como en los reportes anteriormente mencionados, estas diferencias podrían explicarse por las diferentes condiciones ambientales de los lugares de estudio y por la posible prevalencia de las helmintiasis.

Con relación al hallazgo de ooquistes de Cryptosporidium sp., nuestros resultados corroboran las observaciones de Miranda y Martínez (1997) quienes luego de estudiar moscas capturadas en el interior de las viviendas de un pueblo joven del distrito de San Juan de Lurigancho en Lima, obtuvieron $20 \%$ de muestras positivas cuando aplicaron el método de coloración ácido resistente modificado y $15 \%$ por la técnica de Inmunofluorescencia Directa, utilizando anticuerpos monoclonales. Nosotros solo observamos 2 lotes positivos con este parásito y ambas en la misma localidad, donde la población de $M$. domestica fue abundante.

Finalmente, a pesar de que el número de parásitos encontrados en las moscas es reducido, es posible que estos insectos actúen como vectores mecánicos de parásitos, ya sea por las características biológicas de $M$. domesti$c a$, sus hábitos y por haber encontrado muestras positivas con quistes de protozoarios de importancia médica. En consecuencia la mosca doméstica puede ser considerada como un importante transmisor de enfermedades parasitarias en los pueblos jóvenes de la ciudad de Lima.

\section{Agradecimientos}

Al Dr. Manuel Tantaleán V. por las sugerencias y la revisión del manuscrito.

\section{Literatura citada}

Acosta, L. 1997. Prevalencia de Blastocystis hominis (Sarcomastigophora), por el método simple de cultivo en pacientes del Instituto de Salud del Niño. Tesis Facultad de Ciencias Biológicas, Escuela Academico Profesional de Ciencias Biológicas. Universidad Nacional Mayor de San Marcos. Lima, Perú. 62 pp.

Camac, E. 1992. Prevalencia de parasitosis intestinal y factores condicionantes en la jurisdicción Centro de Salud Ganimedes - Distrito de San Juan de Lurigancho. Tesis. Facultad de Medicina. Escuela de Enfermería. Universidad Peruana Cayetano Heredia. Lima, Perú. 46 pp.

Colomina, J. y J. Villar. 1997. Características morfológicas, clínicas y terapéuticas de Cyclospora cayetanensis. Bol. Chil. Parasitol. 52: 26 - 32.

Custodio, M.; y S. N. Murga. 1997. Enteroparásitos transportados por Musca domestica, en Moche, Trujillo - Perú. Libro de Resúmenes. Sociedad Peruana de Parasitología. II Congreso Peruano de Parasitología - Arequipa, pág. 117.

Gamarra, M. y S. Ocampos. 1996. Musca domestica y Blattidae como vectores mecánicos de enteroparásitos en Pueblos Jóvenes del Distrito de Monsefú - Dpto. de Lambayeque 1995. Tesis. Facultad de Biología. Universidad Nacional Pedro Ruiz Gallo. Lambayeque, Perú. 59 pp.

Guillén-de-Tantaleán, Z.; R. Martínez, A. Del aguila, y R. Cusi. 1984. «Moscas» y «cucarachas» como vectores de parasitosis en el Pueblo joven 14,5 Hectáreas - Callao. Boletín Trabajo Extramural. Programa Académico de Medicina Humana Universidad Nacional Mayor de San Marcos. Lima, Perú 1: 13 - 16.

Keiding, J. 1987. La mosca doméstica: Biología y Control. Documento de la Organización Mundial de la Salud. OMS/VBC/86.937, 69 pp.

Manrique, P. y H. Delfin. 1997. Importancia de las moscas como vectores potenciales de enfermedades diarréicas en humanos. Rev. Biomédica 8: $163-170$.

Miranda, E. y R. Martinez. 1997. Estudio preliminar sobre la importancia de las moscas como transmisoras mecánicas de ooquistes de Cryptosporidium sp. Libro de Resúmenes. VI Reunión Científica ICBAR. Facultad de Ciencias Biológicas, Universidad Nacional Mayor de San Marcos p: 84.

Moreno, E. 1980. Biología comparada de Muscidiforax sp y Spalangia endius (Hymenóptera: Pteromalidae) ectoparásitos pupales de la mosca doméstica (Musca domestica). Tesis. Programa Académico de Biología. Universidad Ricardo Palma - Lima, Perú. 72 pp.

OPS, Organización Panamericana de la Salud 1962. Moscas de importancia para la salud pública y su control. Washington, D.C. Publicación Científica $\mathrm{N}_{-}^{\circ} 61,44$ pp.

Tantaleán, M. 1998. Coloración de Protozoarios del tubo digestivo para preparaciones permanentes. Laboratorio de Parasitología. Facultad de Ciencias. Universidad Peruana Cayetano Heredia. 15 pp.

Zarate, A; y Mejia, E. 1995. Frecuencia de enteroparásitos del hombre portados por Musca domestica y Periplaneta americana en Trujillo. Libro de Resúmenes. II Congreso Peruano de Parasitología. Sociedad Peruana de Parasitología. Trujillo, Perú. P: 8. 